\title{
Constraints on the Photon Charge from Observations of Extragalactic Sources
}

\author{
V. V. Kobychev ${ }^{1 *}$ and S. B. Popov ${ }^{2,3^{* *}}$ \\ ${ }^{I}$ Institute for Nuclear Research, National Academy of Sciences of Ukraine, Kiev, Ukraine \\ ${ }^{2}$ University of Padova, Padova, 35122 Italy \\ ${ }^{3}$ Sternberg Astronomical Institute, Universitetskiu pr. 13, Moscow, 119992 Russia
}

Received January 26, 2004

\begin{abstract}
Having analyzed high-resolution observations of extragalactic compact radio sources with modern systems of radio telescopes, we obtained an estimate of the upper limit for the photon electric charge, $e_{\gamma} \lesssim 3 \times 10^{-33}$ of the elementary charge (assuming the photon charge to be energy independent). This is three orders of magnitude better than the limit obtained from radio pulsar timing. We also set a limit on the charge of a gamma-ray ( energy $\sim 0.1 \mathrm{MeV}$ ) photon. In the future, the estimate based on extragalactic sources can be improved significantly. (C) 2005 Pleiades Publishing, Inc.
\end{abstract}

Key words: fundamental constants, radio sources, extragalactic magnetic fields.

\section{INTRODUCTION}

At present, the most stringent upper limit on the photon electric charge is obtained from the timing of Galactic millisecond radio pulsars. Radio pulses are smeared due to the dispersion of charged photons as they move in the interstellar magnetic field (Cocconi 1988; the result was subsequently refined by Raffelt 1994): $e_{\gamma} / e<5 \times 10^{-30}$.

Slightly weaker constraints on the photon charge were discussed by Cocconi (1992) using a different approach based on the angular spread of photons from distant extragalactic sources (which arises from the deviation of a photon with a hypothetical small charge in a magnetic field from rectilinear motion). This method was used to obtain an estimate of $e_{\gamma} / e<$ $10^{-27.7} \approx 2 \times 10^{-28}$ by analyzing the motion of photons in the Galactic magnetic field $\left(B \sim 10^{-6} \mathrm{G}\right.$, a path length of $l \sim 10 \mathrm{kpc}$ ). This bound on the photon charge can be significantly improved primarily by increasing the path length, i.e., when considering the effect in intergalactic fields, and by extending the bandwidth (the Cocconi's limit (1992) is based on observations in a rather narrow bandwidth of $\sim 2 \mathrm{MHz}$ ).

Another constraint has recently been obtained by studying the properties of the cosmic microwave background (CMB). The existence of a small photon charge would result in charge asymmetry of the Universe and would contribute to the observed CMB

*E-mail: kobychev@kinr.kiev.ua

${ }^{* *}$ E-mail: polar@sai.msu.ru anisotropy. Quantitative analysis (Caprini et al. 2003) yields a very stringent upper limit, $e_{\gamma} / e<$ $10^{-38}$, but only if the charge asymmetries produced by different types of particles are not anticorrelated and, more importantly, if the photons have charges of only one sign. These assumptions make this limit model-dependent. ${ }^{1}$

Note that the best laboratory limit, $e_{\gamma} / e<8.5 \times$ $10^{-17}$ (Semertzidis et al. 2003), is much worse than the astrophysical bounds.

\section{CALCULATIONS AND ESTIMATES}

Recent observations have revealed that the magnetic fields in many clusters of galaxies reach significant strengths (several microgauss) with a characteristic autocorrelation length of several kpc (see the review by Carilli and Taylor (2002) and references therein). If the photon had a small, but finite electric charge (with all photons having charges of the same sign), then photons with different energies would move along different trajectories in the intracluster magnetic field. This would cause the angular size of a source to increase (of course, an identical but weaker effect must also exist for intercluster fields). In addition, during observations at two different frequencies, the image centroids would be shifted rela-

${ }^{1}$ See also the previous paper by Sivaram (1994) who discussed the limit based on CMB data. 
tive to one another, ${ }^{2}$ and the photons emitted simultaneously with different energies would reach an observer at different times. If, alternatively, the emitted photons had identical (in absolute value) charges of different signs, the images would be smeared even for monoenergetic radiation. However, the position of the image centroid does not depend on the photon energy in this case.

An ultrarelativistic particle (a photon) with an electric charge $e_{\gamma}$ and a momentum $p=h \nu / c$ in a magnetic field with a component $B_{Y}$ perpendicular to the momentum moves along a trajectory with a radius of curvature $r_{H}$ (the Larmor radius) equal to $c p /\left(e_{\gamma} B_{Y}\right)$. The deviation is thus seen to be largest for low-energy radio photons; furthermore, the angular resolution of VLBI radio observations is much better than that in other parts of the electromagnetic spectrum. Thus, one would expect the best limit on the photon charge to be obtainable in the radio frequency range. However, since the effective photon charge can originate from Lorentz invariance violation, we will also discuss the bounds on the charge for high-energy (of the order of $\mathrm{MeV}$ ) photons.

A photon that travels along an arc of a circumference with a radius $r_{H}$ is deflected through an angle $d l / r_{H}$ (in radians) when traversing a distance $d l$. Therefore, a source at a distance of $l_{\star}$ from the detector will be seen shifted along the $X$ axis perpendicular to the line of sight (the $Z$ axis) by the angle

$$
\varphi_{X}=\int_{0}^{l_{\star}} \frac{d l}{r_{H}(l)}=\frac{e_{\gamma}}{h} \int_{0}^{l_{\star}} \frac{B_{Y}(l) d l}{\nu(l)} .
$$

The $l$ dependence of the frequency appears for cosmological distances due to the redshift: $\nu(z)=$ $(1+z) \nu_{0}$.

Two photons with different energies will diverge by the angle

$$
\Delta \varphi=\varphi_{1}-\varphi_{2}=\frac{e_{\gamma}}{h} \int_{0}^{l_{\star}} B_{Y}\left(\frac{1}{\nu_{1}}-\frac{1}{\nu_{2}}\right) d l
$$

(assuming the photon charge to be energy independent). Thus, the following constraint on the photon charge can be obtained from observations of a source with an angular diameter $\Delta \varphi$ in a band $\Delta \nu$ (with $\Delta \nu \ll \nu)$ :

$$
e_{\gamma} / e \lesssim \frac{\Delta \varphi h}{e}\left(\int_{0}^{l_{\star}} \frac{B_{Y} \Delta \nu d l}{\nu^{2}}\right)^{-1} .
$$

\footnotetext{
${ }^{2}$ In this paper, we assume the angles of deviation to be small.
}

On the other hand, during observations in two widely separated frequency bands $\left(\nu_{1} \ll \nu_{2}\right)$, we may assume that $\Delta \nu / \nu^{2} \approx 1 / \nu_{1}$ :

$$
e_{\gamma} / e \lesssim \frac{\Delta \varphi h}{e}\left(\int_{0}^{l_{\star}} \frac{B_{Y} d l}{\nu_{1}}\right)^{-1}
$$

Here, $\Delta \varphi$ should be considered not as the angular diameter of the source, but as the angular distance between the apparent positions of the source in two different bands. The integrals in Eqs. (3) and (4) can be estimated from observational data on the Faraday rotation of the polarization plane of radio waves that propagate in a magnetized plasma. This quantity can be expressed in terms of the rotation measure, which is defined as the rotation angle of the polarization divided by the wavelength squared. The rotation measure $R M$ can be expressed in terms of the electron density $n_{e}$ on the line of sight and the longitudinal projection of the magnetic field as follows (Clarke et al.2001):

$$
R M=8.12 \times 10^{5} \int_{0}^{l_{\star}} n_{e} B_{Z} d l .
$$

Here, the distance is in Mpc, $n_{e}$ is in $\mathrm{cm}^{-3}$, and $B_{Z}$ is the longitudinal projection of the magnetic field (in microgauss). Assuming the field to be distributed isotropically, without a preferential direction, the longitudinal field projection under the integral can be substituted with the projection onto any other axis, for example, $B_{Y}$. Taking this assumption, below we omit the subscript on $B$.

Above, we disregarded the redshift dependence of the quantities. It would be reasonable to rewrite the formulas using the redshift $z$ rather than the distance as the variable. In addition, the cosmological effects should be taken into account.

The integration element can be written as

$$
d l=-\frac{c}{H_{0}}(1+z)^{-3 / 2} d z
$$

(this equation corresponds to a flat Universe without any dark energy contribution; including this contribution would make the limit more stringent; $H_{0}$ is the Hubble constant $), B(z)=B_{0}(1+z)^{2}, \nu(z)=\nu_{0}(1+$ z) (Ryu et al. 1998).

Equation (3) then transforms to

$$
\begin{gathered}
e_{\gamma} / e<\frac{\Delta \varphi h}{e}\left[\int_{0}^{z_{\star}} \frac{\Delta \nu_{0}(1+z)}{\nu_{0}^{2}(1+z)^{2}}\right. \\
\left.\times B_{0}(1+z)^{2} \frac{c}{H_{0}}(1+z)^{-3 / 2} d z\right]^{-1}
\end{gathered}
$$




$$
=\frac{\Delta \varphi h}{e} \frac{H_{0} \nu_{0}^{2}}{c B_{0} \Delta \nu_{0}} \frac{1}{2\left(\sqrt{1+z_{\star}}-1\right)} \text {. }
$$

For $z_{\star} \ll 1$, the last fraction in Eq. (6) turns into $1 / z_{\star}$.

In most cases, the line-of-sight magnetic field for extragalactic sources is difficult (or impossible) to estimate with a sufficient accuracy. As an illustration, let us obtain a constraint on the photon electric charge using existing estimates of large-scale extragalactic magnetic fields. When considering the effect of a large-scale magnetic field (with scales larger than the size of a cluster of galaxies), one can use the estimate by Kronberg (1994) for the upper limit on the "cosmologically aligned" magnetic field, $B_{0}<10^{-11} \mathrm{G}$ (these data were obtained from the upper bound of $5 \mathrm{rad} \mathrm{m}^{-2}$ on any systematic growth of the rotation measure with distance for $z=2.5$ ), and the upper limit $B_{0}<10^{-9} \mathrm{G}$ for a changing field with a correlation length of $\sim 1 \mathrm{Mpc}$. Widrow (2002) gave the upper limit on the uniform cosmological field component, $\quad B_{0}<6 \times 10^{-12} \mathrm{G}\left(n_{e} / 10^{-5} \mathrm{~cm}^{-3}\right)^{-1}$, which agrees with the above estimate by Kronberg. Various studies (see the review by Widrow 2002) indicate that the actual rotational measure cannot be less than the current upper limit by two or three orders of magnitude. Therefore, for our illustrative estimate, we can conservatively take $B_{0}>6 \times 10^{-15} \mathrm{G}$ as the lower limit for the "noncompensated" cosmological field.

To estimate what constraints on the charge can be obtained for a photon that travels in such a weak field, we will use actual observations. As part of the VSOP (the VLBI Space Observatory Program), Lobanov et al. (2001) observed the quasar PKS 2215+020 at $\nu_{0}=1.6 \mathrm{GHz}$ in a bandwidth of $\Delta \nu_{0}=32 \mathrm{MHz}$. The angular resolution was about 1 mas. The redshift of the source, $z_{\star}=3.57$, corresponds to a distance of $l_{\star}=4700 \mathrm{Mpc}$. Substituting these values into Eq. (6) and taking $B_{0}=6 \times 10^{-15} \mathrm{G}$ and $H_{0}=$ $70 \mathrm{~km} \mathrm{~s}^{-1} \mathrm{Mpc}^{-1}=2.3 \times 10^{-18} \mathrm{~s}^{-1}$, we obtain a bound for the limit on the photon charge,

$$
e_{\gamma} / e \lesssim 6 \times 10^{-29}
$$

which is only an order of magnitude worse than the constraint of Raffelt (1994). However, we used a very conservative lower limit for the noncompensated intergalactic magnetic field on the line of sight. Therefore, as another example, let us consider (at the same frequencies and angular resolution) how this limit would improve if the source were observed through a typical cluster of galaxies (relatively close to us, $z \ll$ $1)$. When the cosmological effects are disregarded, the integral in Eq. (3) transforms into $\left(\Delta \nu / \nu^{2}\right) \int B d l$. If the value of the latter integral for our estimate is taken to be $B l=1 \mu \mathrm{G} \mathrm{Mpc}$ (the product of the typical values of the intracluster field and the size of the central part of the cluster), we then obtain

$$
e_{\gamma} / e<\frac{\Delta \varphi h}{e} \frac{\nu^{2}}{B l \Delta \nu}=2 \times 10^{-33} .
$$

Thus, observing a source through relatively strong intracluster fields (which, in addition, are known with a better accuracy that the fields outside clusters of galaxies) allows us to improve significantly the limit on the photon electric charge at the same angular resolution, although the path length in the field decreases.

\section{A SPECIFIC EXAMPLE}

In the example considered below, the cluster of galaxies used as a scattering screen on the line of sight has $z \ll 1$ (we ignore the influence of the intercluster field). Since the distance dependence of the frequency in Eqs. (3) and (4) may be disregarded at low $z$, we can directly express the limit on the photon charge in terms of the observed rotation measure by eliminating the distribution of the magnetic field and the electron density along the line of sight from the formulas:

$$
e_{\gamma} / e \lesssim 3.2 \times 10^{-19} \frac{\Delta \varphi h}{e} f(\nu)^{-1} \frac{812 h_{70}^{1 / 2}}{R M},
$$

where $f(\nu)=\Delta \nu / \nu^{2}$ or $1 / \nu_{1}$, depending on the relationships between the frequencies. We used Eq. (5) and expressed the electron density as $n_{e}=$ $10^{-3} h_{70}^{1 / 2} \mathrm{~cm}^{-3}$ (Clarke et al. 2001). ${ }^{3}$

For observations in widely separated frequency bands $\left(\nu_{1} \ll \nu_{2}\right)$, this formula can be rewritten as

$$
\begin{gathered}
e_{\gamma} / e \lesssim 1.8 \times 10^{-32} h_{70}^{1 / 2}\left(\frac{\Delta \varphi}{0^{\prime \prime} .001}\right) \\
\times\left(\frac{\nu_{1}}{1 \mathrm{GHz}}\right)\left(\frac{\mathrm{RM}}{1 \mathrm{rad} / \mathrm{m}^{2}}\right)^{-1} .
\end{gathered}
$$

Let us consider the compact radio source 3C 84 in the galaxy NGC 1275 located near the center of the Perseus cluster (Abell 426, $z=0.018$ ). This source was observed by Scott et al. (2004), who surveyed 102 active galactic nuclei at $5 \mathrm{GHz}$ as part of the VSOP project (the VLBI network of telescopes, including the HALCA satellite antenna with an apogee of $21400 \mathrm{~km}$ ). The smallest (in angular size) of the six observed components of 3C 84 has an isophotal diameter at half maximum (FWHM) of 0.8 mas.

\footnotetext{
${ }^{3}$ Note that the Hubble constant $h_{70}$ appears in the formulas only with the subscript that denotes the normalization to $70 \mathrm{~km} \mathrm{~s}^{-1} \mathrm{Mpc}^{-1}$, while the Planck constant $h$ is written everywhere without a subscript.
} 
Since these authors provided the measurement error of the angular diameter, $10 \%$, we conservatively take 0.9 mas as a more reliable estimate of the angular diameter. The central frequency and the bandwidth are $4.8 \mathrm{GHz}$ and $32 \mathrm{MHz}$, respectively.

The rotation measure for 3C 84 was measured by Rusk (1988) (cited by the review of Aller et al. 2003): $\mathrm{RM}=+76 \mathrm{rad} \mathrm{m}^{-2}$. In addition, the Perseus cluster is a source of polarized dispersed radio emission at $350 \mathrm{MHz}$ (Brentjens and de Bruyn 2003) with $R M \sim 25-90 \mathrm{rad}^{-2}$, including the cluster outskirts. Therefore, although part of the rotation measure on the line of sight to 3C 84 was gained in regions with a high electron density at the cluster center, we can safely assume that at least $25 \mathrm{rad} \mathrm{m}^{-2}$ was gained in the outer cluster regions. According to the model of the radial $n_{e}$ distribution in the Perseus cluster (formula (4) from Churazov et al. 2003), the electron density outside the central sphere with a radius of $0.3 \mathrm{Mpc}$ is low $\left(\lesssim 10^{-3} \mathrm{~cm}^{-3}\right)$ and depends weakly on the distance from the cluster center.

Let us first consider the case where the photons have charges of opposite signs. In this case, a point source will be smeared even during observations at a single frequency, so we can use Eq. (8). Substituting $\nu_{1}=4.8 \mathrm{GHz}, R M=25 \mathrm{rad} \mathrm{m}^{-2}$, and $\Delta \varphi=$ 0.9 mas, we obtain a limit on the absolute value of the photon charge,

$$
e_{\gamma} / e \lesssim 3 \times 10^{-33}
$$

For photon charges of the same sign, the broadening of the source results from the photon energy difference, i.e., the finite receiver bandwidth $(\Delta \nu=$ $32 \mathrm{MHz}$ ), and the effect will be weaker. For this case, using $f(\nu)=\Delta \nu / \nu^{2}$, we obtain the following limit from Eq. (7):

$$
e_{\gamma} / e \lesssim 4 \times 10^{-31}
$$

\section{DISCUSSION}

Various methods can be used to set limits on the photon charge. They can be associated with different techniques and observations in different spectral ranges.

VLBI observations of close pairs of sources at several frequencies can give a stringent constraint. In this case, the angular distance between the sources can be measured with a high accuracy: as high as several tens of $\mu$ as (Bartel 2003). Multifrequency observations of two sources with different redshifts (such as those performed by Rioja and Porcas (2000)) can yield important upper limits.

Cocconi (1992) also obtained fairly weak constraints from optical and X-ray data on the angular dispersion: $e_{\gamma} / e<10^{-25.4}$. This author used data on the galactic magnetic fields. The limit can be improved significantly by using currently available data on the intergalactic magnetic fields.

Observations of cosmic gamma-ray bursts (GRBs) with known redshifts (these data were lacking at the time the papers by Cocconi $(1988,1992)$ and Raffelt (1994) were published) cannot seriously compete with pulsar timing data and data on the smearing of radio sources. However, as we noted above, the possible energy dependence of the photon charge makes obtaining limits on the charge over a wide energy range justifiable.

The time delay (the dispersion for gamma-ray photons in the interstellar medium may be disregarded) can be written as (Barbiellini and Cocconi 1987)

$$
\Delta t=\frac{e_{\gamma}^{2} B^{2} l_{\star}^{3}}{24 c E^{2}} .
$$

Here, the delay is calculated relative to the arrival time of photons with energies much higher than $E$, since the delay for them, which is inversely proportional to the square of the energy, is negligible. If the observations are performed in a narrow energy range, $\Delta E \ll E$, then a different formula is applicable:

$$
\Delta t=\frac{e_{\gamma}^{2} B^{2} l_{\star}^{3}}{12 c E^{2}} \frac{\Delta E}{E} .
$$

Both formulas are applicable to photons with charges of the same and different signs.

The duration of the GRB leading edge, which can be shorter than $1 \mathrm{~ms}(\sim 200-250 \mu \mathrm{s}$; Schaefer and Walker 1999), may be taken as an estimate of the maximum time delay.

We then obtain (for $\Delta E / E=0.5$ with the cosmological effects disregarded)

$$
\begin{gathered}
e_{\gamma} / e<5.6 \times 10^{-21} \\
\times\left(\frac{E}{100 \mathrm{keV}}\right)\left(\frac{B}{6 \times 10^{-15} \mathrm{G}}\right)^{-1} \\
\times\left(\frac{\Delta t}{0.1 \mathrm{~ms}}\right)^{1 / 2}\left(\frac{l_{\star}}{1000 \mathrm{Mpc}}\right)^{-3 / 2} .
\end{gathered}
$$

Here, we normalize the magnetic field to the lower limit on the uniform component of the extragalactic field without including the chaotic field component, for which there are no reliable estimates. As in the case considered above, the constraint can be improved significantly if a GRB is observed through a cluster with a known magnetic field. 


\section{CONCLUSIONS}

To summarize, we can say that, at present, observations of extragalactic radio sources yield the most stringent constraints on the absolute value of the photon electric charge, $e_{\gamma} / e \lesssim 3 \times 10^{-33}$ (assuming the photons with unlike charges to be emitted with an equal probability and the photon charge to be energy independent). These constraints can be improved by VLBI observations of close pairs of compact sources at widely separated frequencies through a cluster of galaxies with a known magnetic field, since the accuracy of measuring the angular distance between close sources can reach $10 \mu$ as. In addition, it is highly desirable to use data on several sources; this would allow a limit on the photon charge to be set from statistical analyses. ${ }^{4}$ In the future, with the launch of new spaceborne radio antennas, the angular resolution of observations is likely to increase (Bartel 2003; Fomalont and Reid 2004). Therefore, the most stringent upper limits on the photon charge might be expected precisely from highly accurate observations of extragalactic radio sources.

\section{ACKNOWLEDGMENTS}

We wish to thank A.P. Lobanov, the organizers of the HEA-2003 Conference, and the designers and participants of the project Scientific.Ru. We are grateful to an anonymous referee for several valuable remarks that improved the article.

\section{REFERENCES}

1. M. F. Aller, H. D. Aller, and P. A. Hughes, Astrophys. J. 586, 33 (2003); astro-ph/0211265.

2. G. Barbiellini and G. Cocconi, Nature 329, 21 (1987).

3. N. Bartel, Astron. Latin America, ADeLA Publ. Ser. 1, 35 (2003); astro-ph/0303342.
4. M. A. Brentjens and A. G. de Bruyn, The Riddle of Cooling Flows in Galaxies and Clusters of Galaxies, Charlottesville, VA, USA, May 31-June 4, 2003, Ed. by T. H. Reiprich, J. C. Kempner, and N. Socker, Publ. electronically at http://www.astro.virginia.edu/coolflow/proc.php.

5. C. Caprini, S. Biller, and P. G. Ferreira, hepph/0310066 (2003).

6. C. L. Carilli and G. B. Taylor, Annu. Rev. Astron. Astrophys. 40, 319 (2002); astro-ph/0110655.

7. E. Churazov, W. Forman, C. Jones, and H. Böhringer, Astrophys. J. 590, 225 (2003).

8. T. E. Clarke, P. P. Kronberg, and H. Böhringer, Astrophys. J. 547, L111 (2001).

9. G. Cocconi, Phys. Lett. B 206, 705 (1988).

10. G. Cocconi, Am. J. Phys. 60, 750 (1992).

11. E. Fomalont and M. Reid, astro-ph/0409611 (2004).

12. P. P. Kronberg, Rep. Prog. Phys. 57, 325 (1994).

13. A. P. Lobanov, L. I. Gurvits, S. Frey, et al., Astrophys. J. 547, 714 (2001).

14. G. Raffelt, Phys. Rev. D 50, 7729 (1994); hep$\mathrm{ph} / 9409461$.

15. M. J. Rioja and R. W. Porcas, Astron. Astrophys. 355, 552 (2000); astro-ph/0002097.

16. R. E. Rusk, Ph. D. Thesis (Univ. Toronto, 1988).

17. D. Ryu, H. Kang, and P. L. Biermann, Astron. Astrophys. 335, 19 (1998).

18. B. E. Schaefer and K. C. Walker, Astrophys. J. Lett. 511, L89 (1999).

19. W. K. Scott, E. B. Fomalont, S. Horiuchi, et al., Astrophys. J., Supp. Ser. (2004) (in press); astroph/0407041.

20. Y. K. Semertzidis, G. T. Danby, and D. M. Lazarus, Phys. Rev. D 67, 017701 (2003).

21. C. Sivaram, Am. J. Phys. 63, 1473 (1994).

22. L. M. Widrow, Rev. Mod. Phys. 74, 775 (2002); astro$\mathrm{ph} / 0207240$.

Translated by A. Astakhov

\footnotetext{
${ }^{4}$ The importance of statistics was pointed out by an anonymous referee.
} 